Supporting Information for

\title{
Dynamic permeation method to determine partition coefficients of highly hydrophobic chemicals between polydimethylsiloxane (PDMS) and water
}

\author{
JUNG-HWAN KWON, ${ }^{*}{ }^{\dagger}$ THOMAS WUETHRICH, ${ }^{\dagger}$ PHILIPP MAYER, ${ }^{\ddagger}$ BEATE I. ESCHER ${ }^{\dagger}$
}

${ }^{\dagger}$ Department of Environmental Toxicology (Utox), Swiss Federal Institute of Aquatic Science and Technology (Eawag), Überlandstrasse 133, P.O. Box 611, 8600 Dübendorf, Switzerland and ${ }^{\ddagger}$ National Environmental Research Institute, University of Aarhus, PO Box 358, 4000 Roskilde, Denmark

Corresponding author

phone: +41 44823 5567, fax: +41 44823 5028, and e-mail: Jung-Hwan.Kwon@eawag.ch

Contents:

A) Derivation of the analytical solutions for $C_{\text {PDMs }}$ and $C_{\mathrm{w}}$ (equations 3 and 4 )

B) Derivation of the analytical solutions for equation 7-9

Figure S-1. Extraction time profiles for the conventional shaking method for 2,4,6-trichlorophenol and pentachlorophenol as examples.

Figure S-2. Change in aqueous concentration with respect to stirring time in the partition controlled delivery experiments for (a) 1,2-dichlorobenzene, (b) 1,4-dichlorobenzene, (c) 1,2,3-trichlorobenzene, (d) 1,2,4-trichlorobenzene, (e) 1,3,5-trichlorobenzene, (f) 2,4,5-trichlorotoluene, (g) 1,2,3,5-

tetrachlorobenzene, (h) 1,2,4,5-tetrachlorobenzene, (i) pentachlorobenzene, (j) hexachlorobenzene, (k) naphthalene, (l) acenaphthene, (m) phenanthrene, (n) anthracene, (o) fluoranthene, (p) pyrene, (q) benzo(a)anthracene, (r) chrysene, (s) benzo(a)pyrene. Solid lines represent best-fit using equation 3 and dashed lines represent $95 \%$ confidence intervals of the regression.

Figure S-3. Kinetic data from the ABL permeation method for (a) 1,2,3-trichlorobenzene, (b) 1,2,4trichlorobenzene, (c) 1,3,5-trichlorobenzene, (d) 2,4,5-trichlorotoluene, (e) 1,2,3,5-tetrachlorobenzene, (f) 1,2,4,5-tetrachlorobenzene, (g) pentachlorobenzene, (h) hexachlorobenzene, (i) phenanthrene, (j) anthracene, (k) fluoranthene, (l) pyrene, (m) benzo(a)anthracene, (n) chrysene, (o) perylene, (p) benzo(a)pyrene, (q) dibenzo(a,c)anthracene, (r) dibenzo(a,h)anthracene, (s) benzo(g,h,i)perylene. Solid lines represent best-fit using equation 13.

Figure S-4. Comparisons of $\log K_{\text {PDMSw }}$ in this study with literature values from (a) ref 1, 2, 3, 4 and 8 and (b) ref 10, 12 and 15 in the main text. Solid line represents one-to-one correlation. 
A. Derivation of the analytical solutions for $C_{P D M S}$ and $C_{w}$ (equations 3 and 4 )

$$
\begin{aligned}
& \frac{d C_{w}}{d t}=-k_{a} \frac{V_{P D M S}}{V_{w}} C_{w}+k_{d} \frac{V_{P D M S}}{V_{w}} C_{P D M S} \\
& \frac{d C_{P D M S}}{d t}=k_{a} C_{w}-k_{d} C_{P D M S}
\end{aligned}
$$

Equations (1) and (2) can be written in a matrix form

$\dot{\mathbf{C}}=\mathbf{A C}$

where $\mathbf{A}=\left(\begin{array}{cc}-k_{a} \frac{V_{P D M S}}{V_{w}} & k_{d} \frac{V_{P D M S}}{V_{w}} \\ k_{a} & -k_{d}\end{array}\right)$.

Two eigenvalues $\left(\lambda_{1}\right.$ and $\left.\lambda_{2}\right)$ of the matrix A can be obtained from the quadratic equation

$\left(k_{a} \frac{V_{P D M S}}{V_{w}}+\lambda\right)\left(k_{d}+\lambda\right)-k_{a} k_{d} \frac{V_{P D M S}}{V_{w}}=0$

and they are

$$
\lambda_{1}=0, \lambda_{2}=-\left(k_{a} \frac{V_{P D M S}}{V_{w}}+k_{d}\right)
$$

Eigenvectors are obtained by plugging $\lambda_{1}$ and $\lambda_{2}$ into the original equations (1) or (2). They are $\left(\begin{array}{l}k_{d} \\ k_{a}\end{array}\right)$ for $\lambda_{1}$ and $\left(\begin{array}{c}\frac{V_{P D M S}}{V_{w}} \\ -1\end{array}\right)$ for $\lambda_{2}$.

When $\mathbf{X}$ is a matrix composed of eigenvectors, there exists a time derivative $\mathbf{Z}$, which satisfies

$$
\begin{aligned}
& \dot{\mathbf{C}}=\mathbf{X} \dot{\mathbf{Z}} \\
& \dot{\mathbf{Z}}=\mathbf{D Z}+\mathbf{X}^{-1} \mathbf{h}
\end{aligned}
$$

where $\mathrm{D}$ is a diagonal matrix composed of two eigenvalues and $\mathrm{h}$ is a column vector changing with time, which is zero vector in Equation S.1. Thus, equation S.5 is

$$
\left.\left(\begin{array}{l}
\dot{z}_{1} \\
\dot{z}_{2}
\end{array}\right)=\left(\begin{array}{cc}
0 & 0 \\
0 & -\left(k_{q} \frac{V_{P D M S}}{V_{w}}+k_{d}\right.
\end{array}\right)\right)\left(\begin{array}{l}
z_{1} \\
z_{2}
\end{array}\right)
$$

Solving for $\mathrm{z}_{1}$ and $\mathrm{z}_{2}$,

$z_{1}=c_{1}$

$\left.z_{2}=c_{2} \exp \left(-k_{a} \frac{V_{P D M S}}{V_{w}}+k_{d}\right) t\right)$

where $c_{1}$ and $c_{2}$ are constants.

$C_{w}$ and $C_{P D M S}$ are obtained by plugging equations S.7 and S.8 into S.4. 


$$
\begin{aligned}
& C_{w}=k_{d} c_{1}+c_{2} \frac{V_{P D M S}}{V_{w}} \exp \left[-\left(k_{a} \frac{V_{P D M S}}{V_{w}}+k_{d}\right) t\right] \\
& C_{P D M S}=k_{a} c_{1}-c_{2} \exp \left[-\left(k_{a} \frac{V_{P D M S}}{V_{w}}+k_{d}\right) t\right]
\end{aligned}
$$

From the initial conditions,

$$
\begin{aligned}
& C_{w, 0}=k_{d} c_{1}+c_{2} \frac{V_{P D M S}}{V_{w}} \\
& C_{P D M S}=k_{a} c_{1}-c_{2}
\end{aligned}
$$

$c_{1}$ and $c_{2}$ are

$$
\begin{gathered}
c_{1}=\frac{C_{w, 0}+C_{P D M S, 0} \frac{V_{P D M S}}{V_{w}}}{k_{d}+k_{a} \frac{V_{P D M S}}{V_{w}}} \\
C_{2}=\frac{k_{a} C_{w, 0}-k_{d} C_{P D M S, 0}}{k_{d}+k_{a} \frac{V_{P D M S}}{V_{w}}}
\end{gathered}
$$


B. Derivation of the analytical solutions for equation 7-9

$$
\begin{aligned}
& \frac{d C_{P D M S, \text { donor }}}{d t}=k_{a} C_{w}-k_{d} C_{P D M S, \text { donor }} \\
& \frac{d C_{P D M S, \text { acceptor }}}{d t}=k_{a} C_{w}-k_{d} C_{P D M S, \text { acceptor }} \\
& \frac{d C_{w}}{d t}=-2 k_{a} \frac{V_{P D M S}}{V_{w}} C_{w}+k_{d} \frac{V_{P D M S}}{V_{w}}\left(C_{P D M S, \text { donor }}+C_{P D M S, \text { acceptor }}\right)
\end{aligned}
$$

Equations 7-9 can be written in a matrix form

$$
\dot{\mathbf{C}}=\mathbf{A C}
$$

where $\mathbf{A}=\left(\begin{array}{ccc}-k_{d} & 0 & k_{a} \\ 0 & -k_{d} & k_{a} \\ k_{d} \frac{V_{P D M S}}{V_{w}} & k_{d} \frac{V_{P D M S}}{V_{w}} & -2 k_{a} \frac{V_{P D M S}}{V_{w}}\end{array}\right)$.

The eigenvalues of the matrix $\mathrm{A}$ are

$$
\lambda_{1}=0, \lambda_{2}=-k_{d}, \lambda_{3}=-k_{d}-2 k_{a} \frac{V_{P D M S}}{V_{w}}
$$

The eigenvectors are $\left(\begin{array}{c}k_{a} / k_{d} \\ k_{a} / k_{d} \\ 1\end{array}\right)$ for $\lambda_{1},\left(\begin{array}{c}1 \\ -1 \\ 0\end{array}\right)$ for $\lambda_{2}$, and $\left(\begin{array}{c}-\frac{V_{w}}{2 V_{P D M S}} \\ -\frac{V_{w}}{2 V_{P D M S}} \\ 1\end{array}\right)$ for $\lambda_{3}$.

When $\mathbf{X}$ is a matrix composed of eigenvectors, there exists a time derivative $\mathbf{Z}$, which satisfies

$$
\begin{aligned}
& \dot{\mathbf{C}}=\mathbf{X} \dot{\mathbf{Z}} \\
& \dot{\mathbf{Z}}=\mathbf{D Z}+\mathbf{X}^{-1} \mathbf{h}
\end{aligned}
$$

where $\mathbf{D}$ is a diagonal matrix composed of two eigenvalues and $\mathrm{h}$ is a column vector changing with time, which is zero vector in Equation S.15. Thus, equation S.18 is

$$
\left(\begin{array}{c}
\dot{z}_{1} \\
\dot{z}_{2} \\
\dot{z}_{3}
\end{array}\right)=\left(\begin{array}{ccc}
0 & 0 & 0 \\
0 & -k_{d} & 0 \\
0 & 0 & -k_{d}-2 k_{a} \frac{V_{P D M S}}{V_{w}}
\end{array}\right)\left(\begin{array}{c}
z_{1} \\
z_{2} \\
z_{3}
\end{array}\right)
$$

Solving for $z_{1}, z_{2}$, and $z_{3}$,

$$
\begin{aligned}
& z_{1}=c_{1} \\
& z_{2}=c_{2} \exp \left(-k_{d} t\right)
\end{aligned}
$$


$z_{3}=c_{3} \exp \left[-\left(k_{d}+2 k_{a} \frac{V_{P D M S}}{V_{w}}\right) t\right]$

where $c_{1}, c_{2}$, and $c_{3}$ are constants.

Concentrations are obtained by plugging equations S.20-S.22 into S.17.

$$
\begin{aligned}
& C_{P D M S, \text { donor }}=\frac{k_{a}}{k_{d}} c_{1}+c_{2} \exp \left(-k_{d} t\right)-\frac{V_{w}}{2 V_{P D M S}} c_{3} \exp \left[-\left(k_{d}+2 k_{a} \frac{V_{P D M S}}{V_{w}}\right) t\right] \\
& C_{P D M S, a c c e p t o r}=\frac{k_{a}}{k_{d}} c_{1}-c_{2} \exp \left(-k_{d} t\right)-\frac{V_{w}}{2 V_{P D M S}} c_{3} \exp \left[-\left(k_{d}+2 k_{a} \frac{V_{P D M S}}{V_{w}}\right) t\right] \\
& C_{w}=c_{1}+c_{3} \exp \left[-\left(k_{d}+2 k_{a} \frac{V_{P D M S}}{V_{w}}\right) t\right]
\end{aligned}
$$

The initial conditions are

$$
\begin{aligned}
& C_{P D M S, \text { acceptor }}(t=0)=0 ; \frac{k_{a}}{k_{d}} c_{1}-c_{2}-\frac{V_{w}}{2 V_{P D M S}} c_{3}=0 \\
& C_{P D M S, \text { donor }}(t=0)=C_{0} ; \frac{k_{a}}{k_{d}} c_{1}+c_{2}-\frac{V_{w}}{2 V_{P D M S}} c_{3}=C_{0} \\
& C_{w}(t=0)=0 ; c_{1}+c_{3}=0
\end{aligned}
$$

Solving equations S.26-S.28 for $c_{1}, c_{2}$, and $c_{3}$,

$$
\begin{aligned}
& c_{1}=\frac{C_{0}}{2 K_{P D M S w}+\frac{V_{w}}{V_{P D M S}}} \\
& c_{2}=\frac{1}{2} C_{0} \\
& C_{3}=-\frac{C_{0}}{2 K_{P D M S W}+\frac{V_{w}}{V_{P D M S}}}
\end{aligned}
$$

Substituting S.29-S.31 into S.23-S.25,

$$
\begin{aligned}
& C_{P D M S, \text { donor }}(t)=\left\{\frac{K_{P D M S w}}{2 K_{P D M S w}+\frac{V_{w}}{V_{P D M S}}}+\frac{1}{2} \exp \left(-k_{d} t\right)+\frac{\frac{V_{w}}{2 V_{P D M S}}}{2 K_{P D M S w}+\frac{V_{w}}{V_{P D M S}}} \exp \left[-\left(k_{d}+2 k_{a} \frac{V_{P D M S}}{V_{w}}\right) t\right]\right\} C_{0} \\
& C_{P D M S, a c c e p t o r}(t)=\left\{\frac{K_{P D M S w}}{2 K_{P D M S w}+\frac{V_{w}}{V_{P D M S}}}-\frac{1}{2} \exp \left(-k_{d} t\right)+\frac{\frac{V_{w}}{2 V_{P D M S}}}{2 K_{P D M S w}+\frac{V_{w}}{V_{P D M S}}} \exp \left[-\left(k_{d}+2 k_{a} \frac{V_{P D M S}}{V_{w}}\right) t\right] C_{0}\right. \\
& C_{w}(t)=\frac{C_{0}}{2 K_{P D M S w}+\frac{V_{w}}{V_{P D M S}}}\left\{1-\exp \left[-\left(k_{d}+2 k_{a} \frac{V_{P D M S}}{V_{w}}\right) t\right]\right\}
\end{aligned}
$$


In our experimental condition, $K_{P D M S w}$ is much greater than the volume ratio $\left(\frac{V_{w}}{V_{P D M S}}=5.55\right)$, and thus $2 k_{a} \frac{V_{P D M S w}}{V_{w}}>>k_{d}$. This means than the second exponential terms in equation S.32 and S.33 approach zero much faster than the first exponential term. Therefore, approximations of equations S.32-S.34 are

$$
\begin{aligned}
& C_{P D M S, \text { donor }}(t) \approx \frac{C_{0}}{2}\left[1+\exp \left(-k_{d} t\right)\right] \\
& C_{P D M S, \text { acceptor }}(t) \approx \frac{C_{0}}{2}\left[1-\exp \left(-k_{d} t\right)\right] \\
& C_{w}(t) \approx \frac{C_{0}}{2 K_{\text {PDMS }}}
\end{aligned}
$$




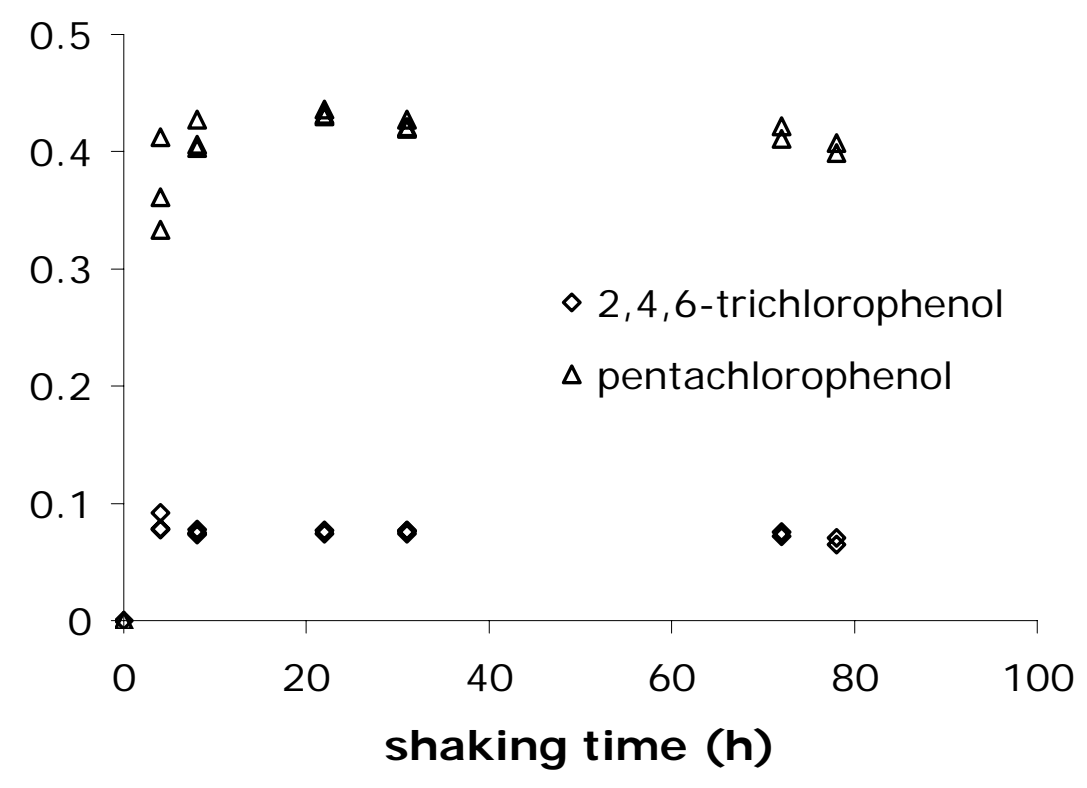

Figure S-1. Extraction time profiles for the conventional shaking method for 2,4,6-trichlorophenol and pentachlorophenol as examples. 

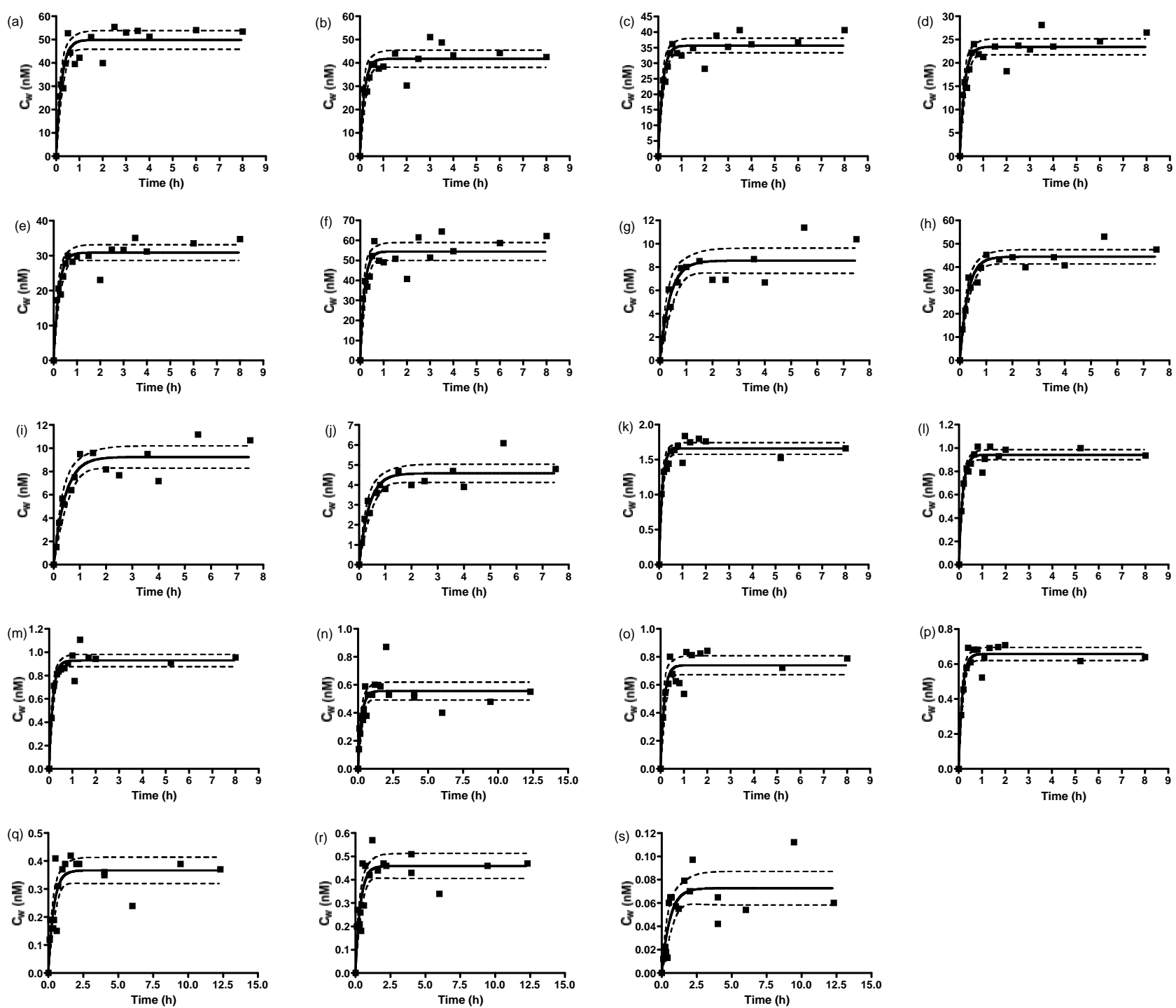

Figure S-2. Change in aqueous concentration with respect to stirring time in the partition controlled delivery experiments for (a) 1,2-dichlorobenzene, (b) 1,4-dichlorobenzene, (c) 1,2,3-trichlorobenzene, (d) 1,2,4-trichlorobenzene, (e) 1,3,5-trichlorobenzene, (f) 2,4,5-trichlorotoluene, (g) 1,2,3,5tetrachlorobenzene, (h) 1,2,4,5-tetrachlorobenzene, (i) pentachlorobenzene, (j) hexachlorobenzene, (k) naphthalene, (l) acenaphthene, (m) phenanthrene, (n) anthracene, (o) fluoranthene, (p) pyrene, (q) benzo(a)anthracene, (r) chrysene, (s) benzo(a)pyrene. Solid lines represent best-fit using equation 3 and dashed lines represent 95\% confidence intervals of the regression. 

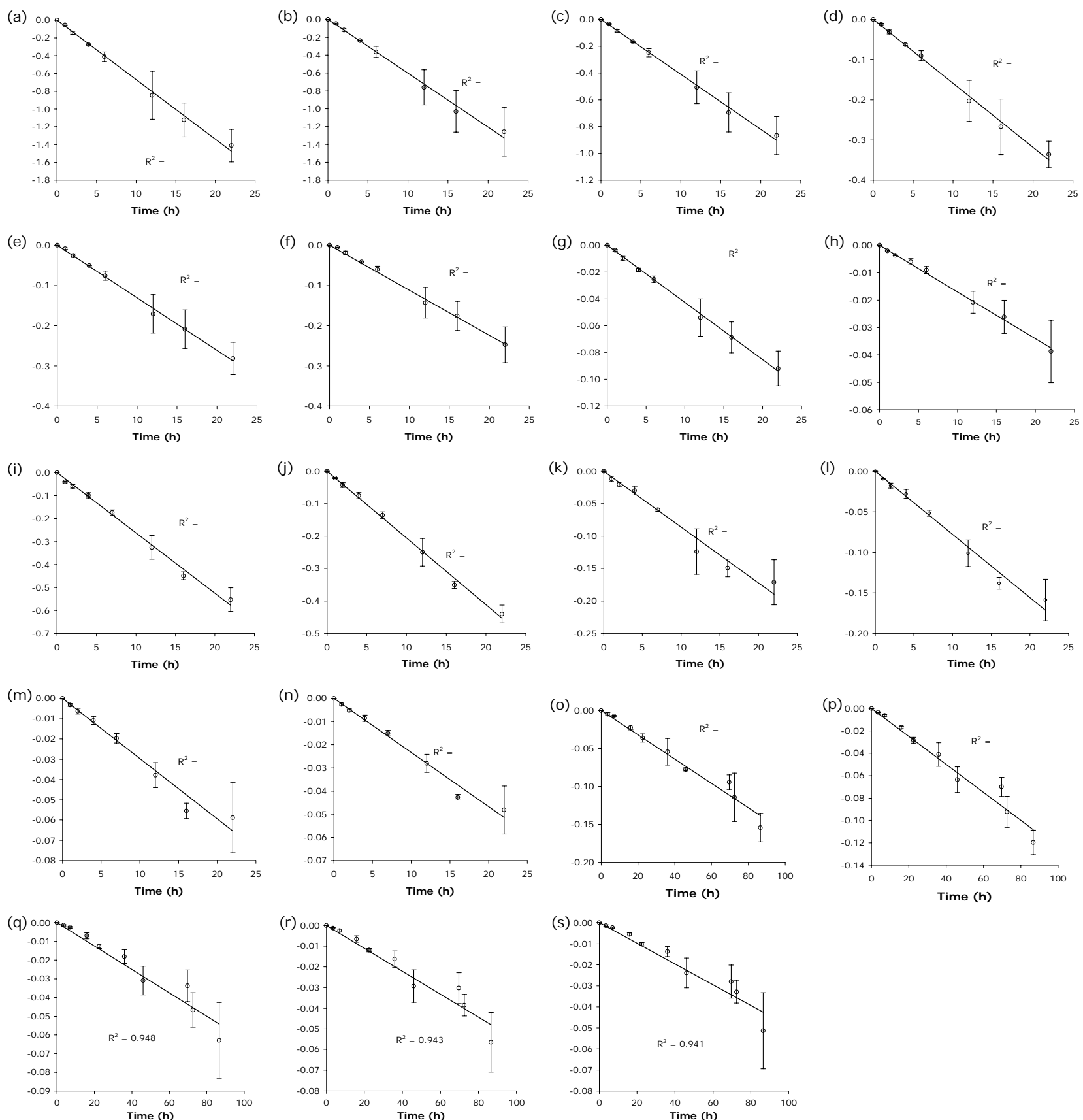

Figure S-3. Kinetic data from the ABL permeation method for (a) 1,2,3-trichlorobenzene, (b) 1,2,4trichlorobenzene, (c) 1,3,5-trichlorobenzene, (d) 2,4,5-trichlorotoluene, (e) 1,2,3,5-tetrachlorobenzene, (f) 1,2,4,5-tetrachlorobenzene, (g) pentachlorobenzene, (h) hexachlorobenzene, (i) phenanthrene, (j) anthracene, (k) fluoranthene, (l) pyrene, (m) benzo(a)anthracene, (n) chrysene, (o) perylene, (p) benzo(a)pyrene, (q) dibenzo(a,c)anthracene, (r) dibenzo(a,h)anthracene, (s) benzo(g,h,i)perylene. Solid lines represent best-fit using equation 13. 
(a)

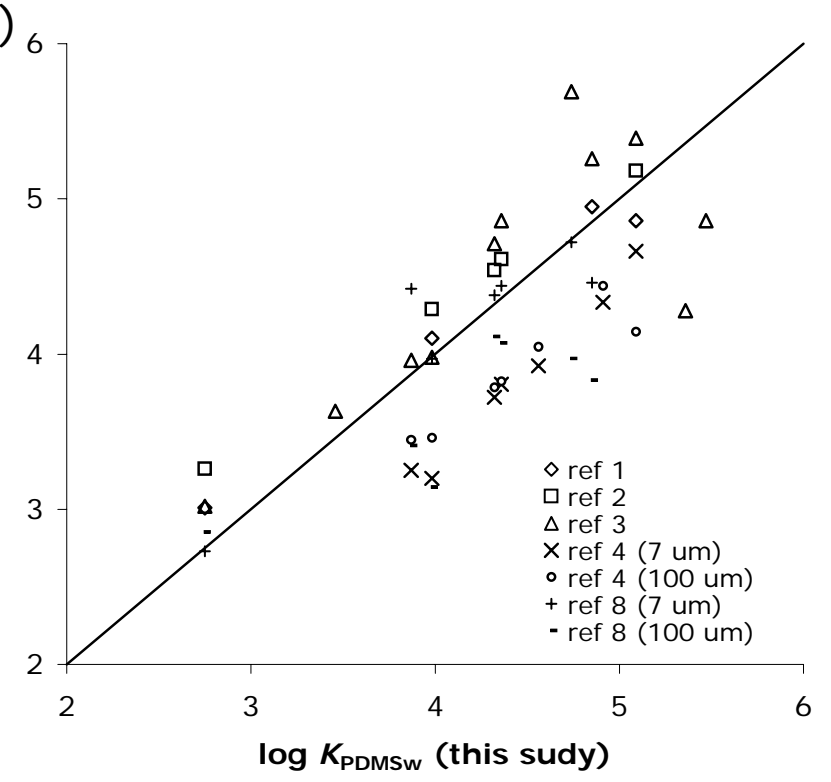

(b)

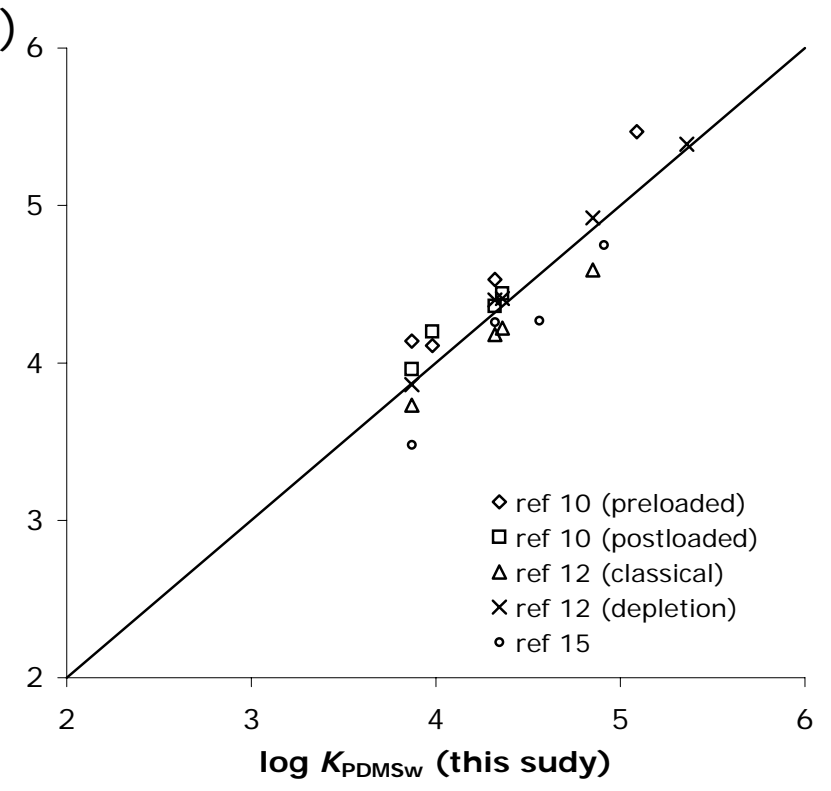

Figure S-4. Comparisons of $\log K_{\text {PDMSw }}$ in this study with literature values from (a) ref 1, 2, 3, 4 and 8 and (b) ref 10, 12 and 15 in the main text. Solid line represents one-to-one correlation. 